\title{
Validação experimental de modelos matemáticos para a predição do volume e área superficial de ovos
}

\author{
Damasceno, F.A. '; Bertoldo, B. ${ }^{2}$; Oliveira, J.L. ${ }^{2}$; Oliveira, C.E.A. ${ }^{2}$; Schiassi, L. ${ }^{1}$ e Silva, A.R. ${ }^{2}$
}

'Departamento de Engenharia. Universidade Federal de Lavras (UFLA). Lavras-MG. Brasil.

${ }^{2}$ Departamento de Engenharia Agrícola e Ambiental. Universidade Federal de Mato Grosso (UFMT). Rondonópolis-MT. Brasil.

\section{PalaVRas ChaVe ADICIONAIS}

Avicultura.

Geometria.

Modelagem matemática.

Produção de ovos.

\section{RESUMO}

Em razão da grande importância das características geométricas do ovo na agroindústria, objetivou-se, com o presente estudo, validar experimentalmente diversos modelos matemáticos do volume e área superficial de ovos, utilizando equações empíricas ajustadas e da literatura, visando a melhoria na precisão do cálculo destes parâmetros. Para isso, 450 amostras de ovos foram coletadas, sendo 150 ovos brancos, 150 ovos vermelhos e 150 ovos de codorna. Cada ovo foi pesado e suas dimensões (comprimento e largura) foram medidas utilizando um paquímetro digital. O volume real de cada ovo foi determinado pelo método de imersão em água. A área superficial, bem como o volume, das amostras foram obtidos por equações empíricas ajustadas, método computacional e por meio de equações da literatura. Com base nos resultados, os ovos vermelhos possuem peso, volume e área superficial estatisticamente maiores em relação os ovos brancos e de codorna $(p<0,05$; Scott-Knott). Todos os modelos para estimativa do volume e da área superficial de ovos, propostos neste trabalho e oriundos da literatura, apresentaram valores médios próximos ao real. A análise de imagem dos ovos, que é não invasiva e não destrutiva adequada, é uma boa alternativa aos métodos destrutivos convencionais.

\section{Experimental validation of mathematical models to predict the volume and surface area of the eggs}

\section{SUMMARY}

Due to magnitude of geometric characteristics of the egg in agribusiness, this research was performed aiming to experimentally validate various mathematical models of volume and surface area of eggs, using adjusted and literature empirical equations in order to improve the accuracy of calculation of these parameters. For this reason, 450 samples of eggs were collected: 150 chicken eggs (white), 150 chicken eggs (brown) and 150 quail eggs. Each egg was weighed and its dimensions (length and width) were measured using a digital caliper. The real volume of each egg was determined by immersion in water. The surface area and volume, the samples were obtained using adjusted empirical equations, and from computational methods and the literature. Based on the results, the brown eggs have weight, volume and surface area significantly higher than white eggs and quail eggs ( $p<0.05$; Scott Knott). Overall, all models to estimate the volume and surface area of eggs, proposed in this paper and from published research, showed mean values close to real. The image analysis eggs, which is neither invasive nor destructive, a good alternative to the conventional destructive methods.

\section{INFORMACIÓN}

Cronología del artículo.

Recibido/Received: 10.2.2015

Aceptado/Accepted: 15.6.2015

On-line: 16.9 .2015

Correspondencia a los autores/Contact e-mail:

leonardo.schiassi@deg.ufla.br

\section{INTRODUÇÃO}

Durante os últimos anos, a avicultura tem investido constantemente em inovações tecnológicas, permitindo novos conceitos e sistemas de criação de aves. Na decisão de implementação desses sistemas, existe a procura por maior eficiência na produção, que tem como pilares a viabilidade econômica e téc- nica, com ênfase nos aspectos produtivos, sanitários e bem-estar das aves (Abreu and Abreu, 2011).

A tecnologia aplicada ao processo avícola, seja por equipamentos ou sistemas monitoradores, tem garantido maior rendimento e produtividade, com qualidade, a custos compatíveis com o mercado (Malavazzi, 1995). 
No Brasil, os ovos são classificados em grupos, classes e tipos, de acordo com a coloração da casca, qualidade e peso (Xavier et al., 2008). Deste modo, ovos de aves de postura tornam-se objetos adequados para investigação em diversas áreas da agricultura e bio-indústrias, devido principalmente pela relativa facilidade da coleta dos ovos, prazo limitado da postura e da agilidade de medição das variáveis em estudo. Ao mesmo tempo, a alta variabilidade dos parâmetros geométricos dos ovos pode criar dificuldade em sua descrição.

Deste modo, o conhecimento dos parâmetros geométricos de um ovo de ave é relevante para uma série de estudos, como a biologia das aves, taxonomia, classificação, ecologia, reprodução, seleção de aves, genética e processamento (Narushin, 1997). Durante alguns estudos envolvendo modelagem matemática e simulação computacional, muitas vezes é necessário determinar alguns parâmetros relacionados à geometria dos ovos, tais como comprimento, largura, volume, área superficial, dentre outros.

Existem vários estudos referentes a modelos matemáticos envolvendo a geometria do ovo (Preston, 1953; Bonnet and Mongin, 1965; Smart, 1967; Rehkugler, 1973; Narushin, 1993; Narushim, 2005; Silva, 2014). Entretanto, segundo Romanoff and Romanoff (1949), é impossível expressar o contorno de ovos individuais em termos matemáticos. Por outro lado, muitos autores tentaram descrever a forma de ovo, por meio de equações matemáticas. As maiorias destes estudos assumiram que a forma do ovo era igual a um elipsóide.

Conforme foi observado por Narushin (1993), é possível transformar a forma de ovo para o contorno de uma elipse, desde que o comprimento do eixo longitudinal e a circunferência longitudinal do ovo e da elipse são iguais uns aos outros. Assim, de acordo com esse autor, a partir de uma descrição matemática da forma de um ovo, é possível deduzir o volume e a área de superfície do ovo, desde que se assuma que o ovo seja um sólido de revolução em torno do eixo longitudinal. A área de superfície do ovo também poderá ser estimada a partir da massa ou volume.

Sendo assim, o problema da avaliação da geometria do ovo pode ser resolvido de duas maneiras: a) por equações matemáticas e, b) em diferentes indicadores que mostram o desvio de uma forma verdadeira de algum objeto utilizado como modelo.

A determinação da área superficial e volume de ovos de aves são importantes para avaliar a capacidade de eclosão, prever a fertilidade, inferir sobre a qualidade da casca, calcular as transferências de calor e massa entre o ovo e o ambiente circundante, dar suporte ao processo de fabricação de bandejas para acomodação dos ovos no armazenamento e inferir sobre o sistema de classificação e industrialização (Narushin, 2005; Carioca et al., 2010; Carvalho and Fernandes, 2013; Silva, 2014).

Em razão da grande importância das características geométricas do ovo na agroindústria, objetivou-se, com o presente estudo, validar experimentalmente diversos modelos matemáticos do volume e área superficial de

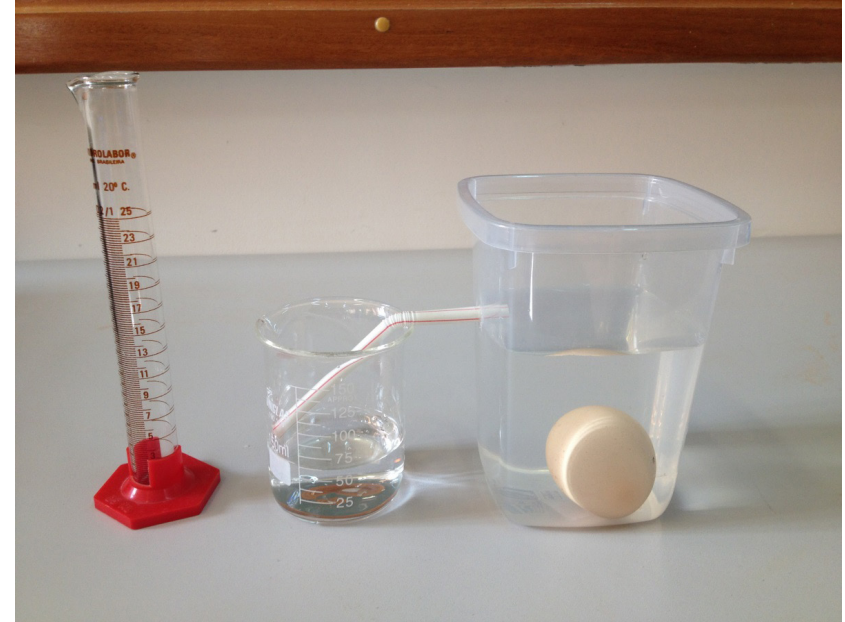

Figura 1. Determinação do volume real do ovo pelo método de imersão em água (Determining the actual egg volume by immersion in water).

ovos, utilizando equações empíricas ajustadas e da literatura, visando à melhoria na precisão do cálculo destes parâmetros.

\section{MATERIAIS E MÉTODOS}

\section{Caracterização dos GalPÕES E COlETA de dAdOS}

Para realização deste estudo, os ovos foram coletados em galpões para criação de aves de postura em um complexo de produção de ovos situado no município de Primavera do Leste - MT (15 $33^{\circ} \mathrm{S}$ de latitude e $54^{\circ} 17^{\prime} \mathrm{O}$ de longitude, $660 \mathrm{~m}$ de altitude).

O complexo produtivo de ovos branco e vermelho possuia 48 galpões com dimensões de $14 \times 130 \mathrm{~m}$, sendo que todos os galpões alojavam aproximadamente 102 mil aves. Os galpões possuiam cobertura de telhas metálicas (espessura de 0,4 mm), alvenarias do tipo tijolo furado $(0,12 \times 0,20 \times 0,20 \mathrm{~m})$, muretas laterais de $0,60 \mathrm{~m}$ de altura, piso de concreto, tela de arame para fechamento lateral de malha de $0,03 \mathrm{~m}$, cortinas laterais de cor azul e sem uso de forro. Os galpões eram orientados na direção leste-oeste e possuíam grama como vegetação circundante. Cada galpão possuía quatro fileiras com seis andares de gaiolas com dimensão de $0,76 \times 0,59 \times 0,50 \mathrm{~m}$ (largura $\times$ profundidade $\times$ altura) e contendo 14 aves da linhagem Hy-Line W-36. Todas as gaiolas dispunham de bebedouros tipo nipple e comedouro do tipo calha em chapa galvanizada e uma esteira automática para recolhimento dos ovos. Abaixo das gaiolas, uma esteira recolhia os dejetos e ovos quebrados, conduzindo para o setor de compostagem da granja.

No caso dos ovos de codornas, as aves foram alojadas em gaiolas individuais, com dimensões de $0,11 \times 0,20 \times 0,16 \mathrm{~m}$ (largura x profundidadexaltura), localizadas no galpão de coturnicultura, que possui 7,0 m de largura $\times$ 40,0 m de comprimento, com cobertura de telha de fibrocimento, piso concretado e paredes laterais de $0,30 \mathrm{~m}$ de altura, e o restante com tela de arame até o telhado, provida de cortinas laterais. Os bebedouros foram do tipo nipple, e o comedouro do tipo calha, disposto na frente das gaiolas. A ração 
foi fornecida à vontade e o programa de iluminação utilizado foi iluminação natural + artificial, totalizando $18 \mathrm{~h}$ por dia.

Foram coletados 450 amostras de ovos, sendo 150 ovos brancos, 150 ovos vermelhos e 150 ovos de codorna. Cada ovo foi pesado e suas dimensões (comprimento e largura) foram medidas utilizando um paquímetro digital.

\section{DETERMINAÇÃO dO VOLUME E ÁREA SUPERFICIAL REAL}

O volume real de cada ovo foi determinado pelo método de imersão em água, onde se mergulhou cada ovo em um recipiente de $500 \mathrm{~mL}$ com água, sendo que o volume deslocado de água foi coletado por um béquer e medido numa proveta de $20 \mathrm{~mL}$. O volume de água deslocado representou o volume real de cada ovo (figura 1).

A área superficial, bem como o volume, das amostras foram obtidos por um método computacional, onde fotos em diferentes posições eram produzidas de cada ovo utilizando uma câmera digital (14.0 Megapixels). Para isso, construiu-se um dispositivo de madeira para fixação desta câmera, objetivando a redução de distorções das imagens causadas devido à diferença de distância focal, garantindo uma melhor qualidade da imagem e posição mais uniforme dos ovos (figura 2).

Em seguida, importaram-se as imagens para o programa AutoCAD ${ }^{\circledR} 2013$ (Autodesk) e ajustaram-se as imagens digitais na escala 1:1 por meio da aplicação
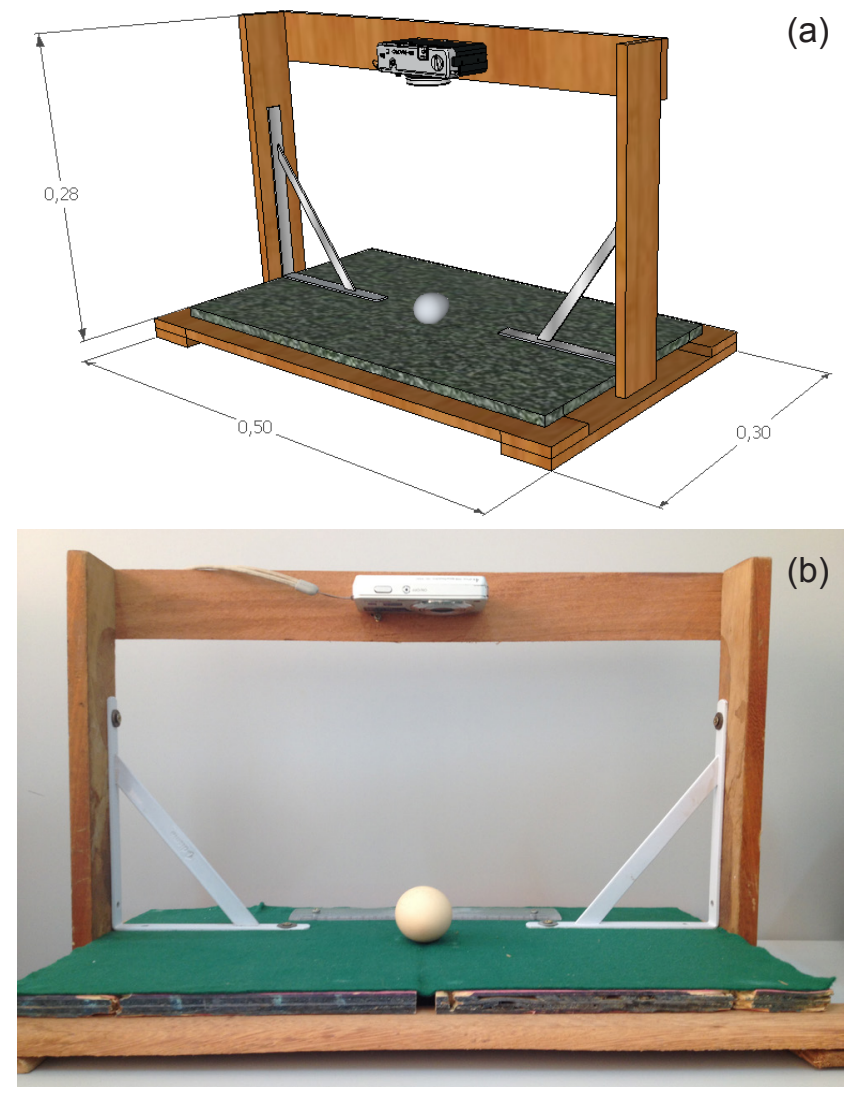

Figura 2. Desenho esquemático em 3D com as principais dimensões (a) e (b) dispositivo para captura das imagens (3D model design with main dimensions (a) and (b) Apparatus to capture of the Images).
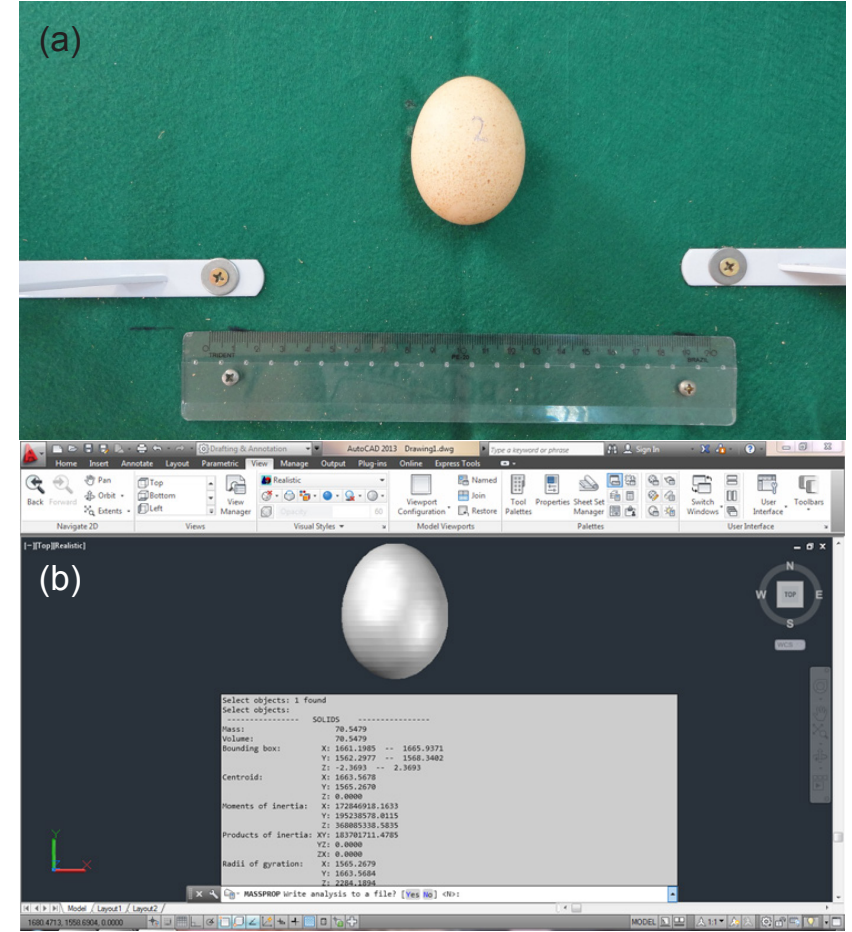

Figura 3. Procedimento para representação tridimensional da imagem de um ovo. Medidas do objeto real (a) e medidas através do programa computacional (b) (Procedure for three-dimensional representation of the image of an egg. Measures of the real object (a) and measured by the computer program (b)).

do comando Scale. Usou-se a média de cinco repetições das medições com o propósito de reduzir os erros durante a coleta de dados. Logo em seguida, contornaram-se as imagens das amostras com uma Polyline, as quais foram divididas em dois planos imaginários perpendiculares entre si. Assim, aplicou-se o comando Rotate, transformando os contornos das imagens digitais em figuras tridimensionais (figura $\mathbf{3 b}$ ). Os valores do volume e área superficial dos ovos pelo método computacional foram adquiridos aplicando-se o comando Properties nas imagens tridimensionais.

\section{MOdelo matemátICO}

Segundo Narushim (1997), o cálculo geométrico do ovo inclui a estimativa do volume (V) e da área superficial (As), que são ainda baseados nas medidas do comprimento máximo (C) e da largura máxima (L).

Deste modo, utilizando o modelo apresentado por Narushin (2005) o resultado da equação para cálculo do $\mathrm{V}$ e As de ovos pode ser obtido pelas equações $1 \mathrm{e}$ 2 , respectivamente:

$$
\begin{aligned}
& V=\left(0,525 \times C \times L^{2}\right) \\
& A s=\left(0,9658 \times \frac{L}{C}+2,1378\right) \times C \times L
\end{aligned}
$$

\section{DELINEAMENTO EXPERIMENTAL}

Neste estudo, procedeu-se à análise de variância e ao teste de médias de Scott-Knott ao nível de 5\% de probabilidade para avaliar as características geométricas dos ovos. 
Tabela I. Dados das medidas geométricas e peso dos diferentes tipos de ovos (Data for geometric measures and weight of different types of eggs).

\begin{tabular}{|c|c|c|c|c|c|}
\hline Tipo de ovo & $\begin{array}{l}\text { Valor } \\
\text { máximo }\end{array}$ & $\begin{array}{l}\text { Valor } \\
\text { mínimo }\end{array}$ & Média & $\begin{array}{l}\text { Desvio } \\
\text { padrão }\end{array}$ & $\begin{array}{c}\text { Coef. de } \\
\text { variação }(\%)\end{array}$ \\
\hline \multicolumn{6}{|c|}{ Peso (g) } \\
\hline Ovo branco & $62,8^{b}$ & $49,0^{b}$ & $55,6^{b}$ & $\pm 3,0$ & 5,4 \\
\hline Ovo vermelho & $67,6^{\mathrm{c}}$ & $52,6^{c}$ & $59,4^{c}$ & $\pm 3,2$ & 5,3 \\
\hline Ovo codorna & $12,8^{\mathrm{a}}$ & $7,0^{\mathrm{a}}$ & $10,5^{\mathrm{a}}$ & $\pm 0,9$ & 8,8 \\
\hline \multicolumn{6}{|c|}{ Largura (mm) } \\
\hline Ovo branco & $56,3^{c}$ & $39,7^{b}$ & $42,4^{b}$ & $\pm 1,4$ & 3,2 \\
\hline Ovo vermelho & $46,1^{\text {b }}$ & $40,7^{b}$ & $43,3^{b}$ & $\pm 1,3$ & 2,9 \\
\hline Ovo codorna & $26,7^{\mathrm{a}}$ & $22,8^{\mathrm{a}}$ & $24,8^{\mathrm{a}}$ & $\pm 0,8$ & 3,0 \\
\hline \multicolumn{6}{|c|}{ Comprimento (mm) } \\
\hline Ovo branco & $61,3^{b}$ & 41,8 & $56,2^{b}$ & $\pm 2,7$ & 4,8 \\
\hline Ovo vermelho & $62,5^{\mathrm{b}}$ & 43,3 & $56,4^{b}$ & $\pm 2,0$ & 3,5 \\
\hline Ovo codorna & $34,3^{\mathrm{a}}$ & $24,6^{\mathrm{a}}$ & $31,5^{\mathrm{a}}$ & $\pm 1,7$ & 5,3 \\
\hline \multicolumn{6}{|c|}{ Volume $\left(\mathrm{cm}^{3}\right)$} \\
\hline Ovo branco & $61,9^{b}$ & $49,5^{b}$ & $55,3^{b}$ & $\pm 2,5$ & 4,6 \\
\hline Ovo vermelho & $68,1^{\mathrm{c}}$ & $48,8^{b}$ & $58,5^{c}$ & $\pm 5,1$ & 8,8 \\
\hline Ovo codorna & $12,7^{a}$ & $7,1^{\mathrm{a}}$ & $10,3^{a}$ & $\pm 1,0$ & 9,9 \\
\hline
\end{tabular}

As médias seguidas de letras iguais, na mesma coluna, não diferem estatisticamente entre si, pelo teste Scott-Knott (5\%).

Com base nos pares de dados de volume e área superficial dos ovos, determinados pelos métodos de imersão, método computacional e equações da literatura, ajustou-se modelos empíricos por meio da subrotina PROC REG do SAS ${ }^{\circledast}$ (SAS, 2001). A significância dos modelos e de cada coeficiente das equações foi avaliada pelos testes $\mathrm{F}$ e $\mathrm{t}$, respectivamente. Para validação, os resultados dos modelos empíricos foram comparados aos medidos por meio do teste $t$.

\section{RESULTADOS E DISCUSSÃO}

Os resultados das medidas geométricas iniciais e do volume dos ovos pelo método de imersão são apresentados na tabela I. Existe pelo menos uma diferença significativa entre os valores médios do peso e volume dos ovos ( $p<0,05$; Scott-Knott). Assim, os ovos vermelhos possuem peso e volume estatisticamente maiores em relação os outros tipos de ovos. Fato que, provavelmente, ocorreu devido a diferenças nos genótipos de raças e idades das aves avaliadas neste estudo. Os ovos brancos e vermelhos apresentaram valores médios de largura e comprimento estatisticamente iguais ( $p>0,05$; Scott-Knott).

Silva et al. (2011), ao estudarem recuperação da topografia de ovos por meio da técnica de Moiré, verificaram que os valores médios do comprimento e largura dos ovos foram aproximadamente 57,0 e 45,2 $\mathrm{mm}$, respectivamente.

Silva (2014), averiguando o volume médio dos ovos de galinhas pelo método de imersão em água, encontrou o valor médio de $50,5 \mathrm{~cm}^{3}$, para os ovos do tipo grande. Narushin et al. (2004), examinando as características geométricas de ovos em duas linhagens diferentes de aves encontraram valores médios de
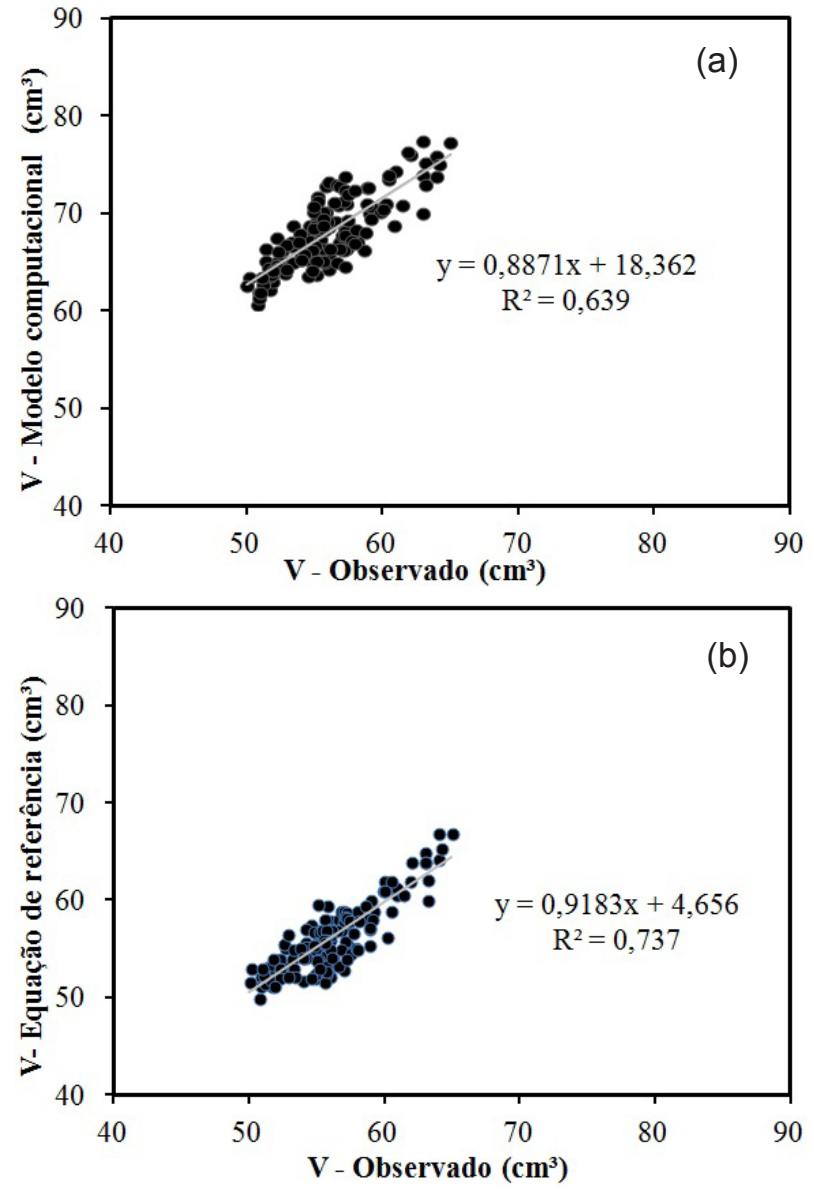

Figura 4. Volume dos ovos brancos observados, estimados pelo modelo computacional (a) e (b) pela equação de referência (Volume of the observed white eggs, estimated by computational model (a) and (b) by reference equation)

peso e volume iguais a $67,17 \mathrm{~g}$ e $62,31 \mathrm{~cm}^{3}$. Narushin (2001), averiguando a forma geométrica de ovos de aves encontrou valores médios do peso, comprimento e volume iguais a $64,4 \mathrm{~g}, 58,5 \mathrm{~mm}$ e $59,6 \mathrm{~cm}^{3}$, respectivamente.

$\mathrm{Na}$ figura 4, ilustra-se as relações funcionais entre os valores de volume de ovos brancos estimados pelo método computacional e por meio de equações da literatura (equações 1 e 2) em função daquelas estimadas pelo método de imersão em água considerada como referência neste trabalho. Por meio de uma análise estatística preliminar, verificou-se que existe diferença estatística significativa entre os volumes dos ovos brancos observados, estimados pelo modelo computacional e pela equação de referência (teste $t, p>0,05$ ). Desta forma, ajustou-se equações para determinação do volume do modelo computacional e da equação de referência em função do volume observado (figura 4). As equações ajustadas para o volume dos ovos brancos estimado pelo modelo computacional apresentou coeficiente de determinação $\left(R^{2}\right)$ de 0,639 em relação ao volume observado, enquanto que o volume estimado pela equação de referência em relação ao volume observado obteve coeficiente de determinação $\left(\mathrm{R}^{2}\right)$ de 0,737 , sendo que, os coeficientes lineares e angulares são significativos (teste $t, p>0,05$ ). Os valores médios e os desvios padrões dos volumes dos ovos 

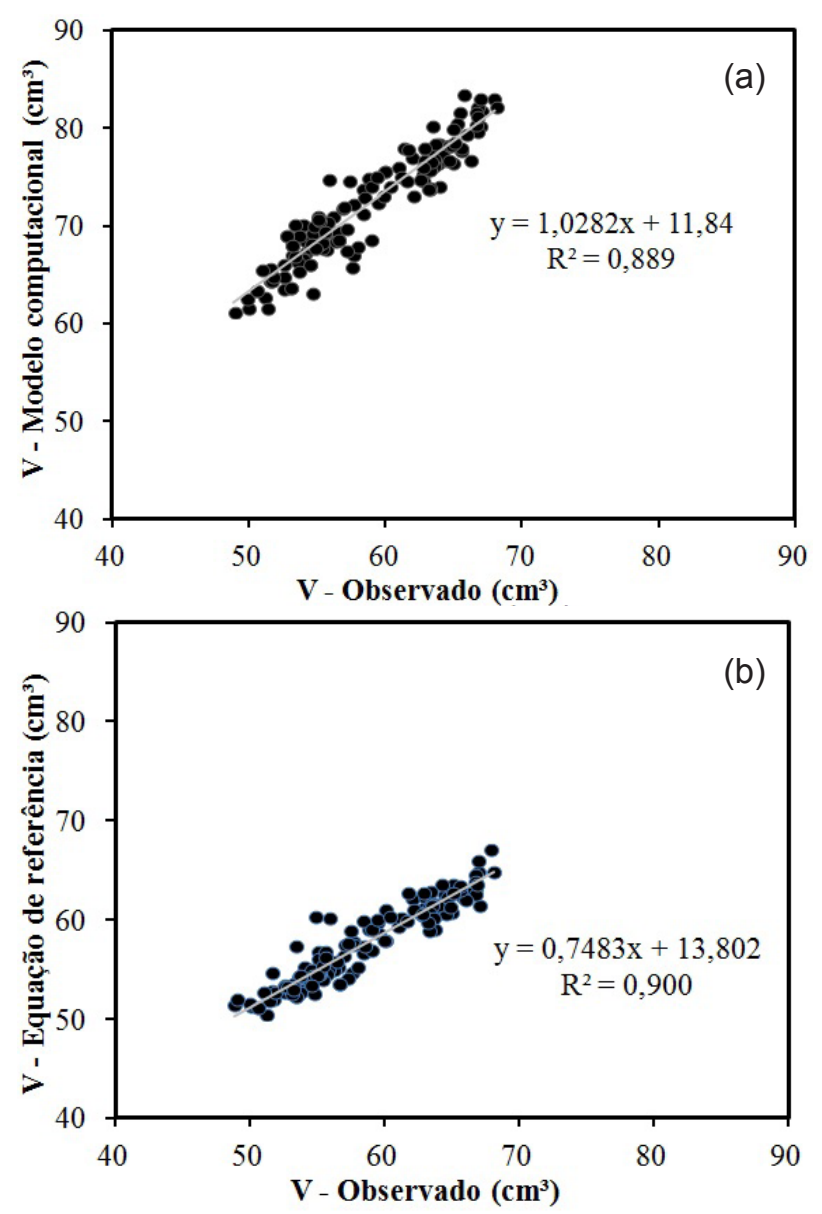

Figura 5. Volume dos ovos vermelhos observados, estimados pelo modelo computacional (a) e (b) pela equação de referência (Volume of the observed red eggs, estimated by computational model (a) and (b) by reference equation).

brancos observados, simulados pelo modelo computacional e calculados pela equação de referência foram de $55,91 \pm 3,22 \mathrm{~cm}^{3}, 67,96 \pm 3,57 \mathrm{~cm}^{3}$ e $56,00 \pm 3,44 \mathrm{~cm}^{3}$, respectivamente.

Na figura 5, podem ser observadas as relações funcionais entre os valores de volume de ovos vermelhos estimados pelo método computacional e por meio de equações da literatura (equações 1 e 2) em função daquelas estimadas pelo método de imersão em água considerada como referência neste trabalho. Conforme pode ser observado pela figura 5, o volume determinado pelo modelo computacional e da equação de referência dos ovos vermelhos apresentaram coeficientes de determinações $\left(R^{2}\right)$ de 0,889 e 0,900 , respectivamente, em relação ao volume observado, sendo que, os coeficientes lineares e angulares são significativos (teste $t, p>0,05)$. Os valores médios e os desvios padrões dos volumes dos ovos vermelhos observados, simulados e calculados foram de 58,52 $\pm 5,15 \mathrm{~cm}^{3}, 72,08 \pm 5,57 \mathrm{~cm}^{3} \mathrm{e}$ $57,58 \pm 4,07 \mathrm{~cm}^{3}$, respectivamente.

Pela figura 6, observam-se as relações funcionais entre os valores de volume de ovos de codorna estimados pelo método computacional e por meio de equações da literatura (equações 1 e 2) em função daquelas estimadas pelo método de imersão em água considerada como referência neste trabalho. As equações ajustadas
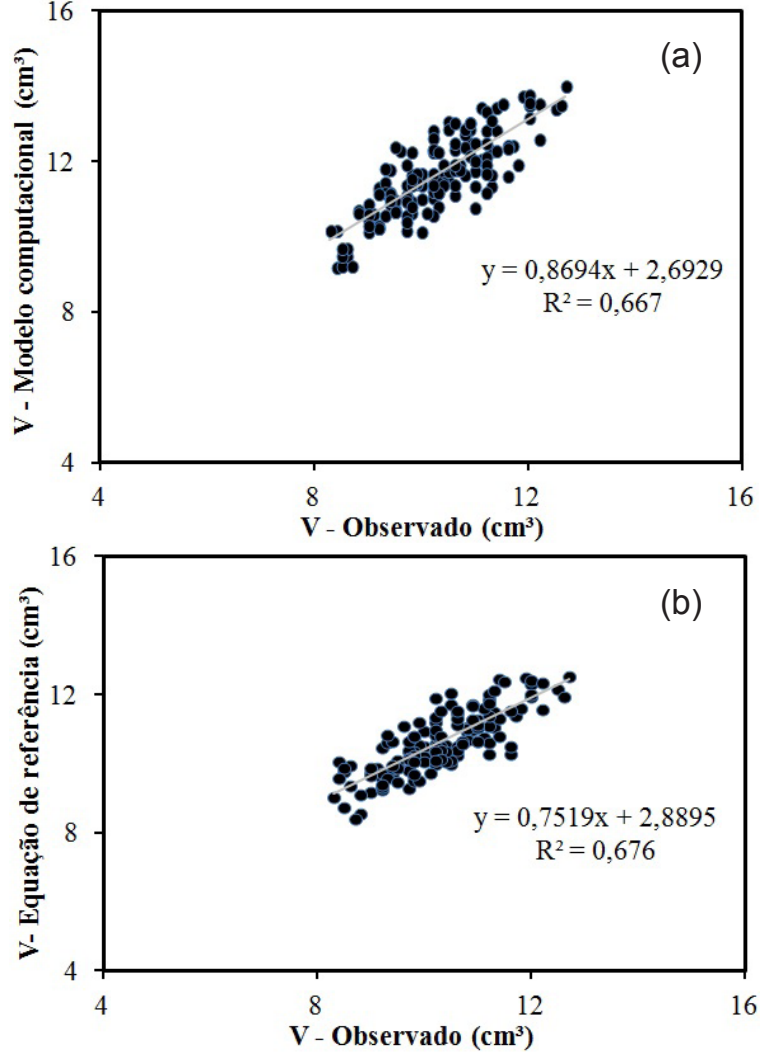

Figura 6. Volume dos ovos de codornas observados, estimados pelo modelo computacional (a) e (b) pela equação de referência (Volume of the observed quail eggs, estimated by computational model (a) and (b) by reference equation).

para os ovos de codorna apresentaram $R^{2}$ de 0,667 em relação ao volume do modelo computacional e volume observado e 0,676, com relação ao volume da equação de referência quando comparada ao volume observado (figura 6), sendo que, os coeficientes linear e angular são significativos (teste $t, p>0,05$ ). Os valores médios e os desvios padrões dos volumes dos ovos vermelhos observados, determinados pelo modelo computacional e calculados pela equação de referência foram de $10,28 \pm 0,99 \mathrm{~cm}^{3}, 11,63 \pm 1,05 \mathrm{~cm}^{3}$ e $10,62 \pm 0,90 \mathrm{~cm}^{3}$, respectivamente.

$\mathrm{Na}$ figura 7, apresenta-se a relação funcional das áreas superficiais observadas e do modelo computacional dos ovos brancos, vermelhos e de codorna, sendo ajustada uma equação para estimar a área superficial observada de cada tipo de ovo. As equações ajustadas apresentaram $\mathrm{R}^{2}$ de 0,630 para ovos brancos, 0,883 para ovos vermelhos e 0,791 para ovos de codorna, sendo que os coeficientes linear e angular são significativos (teste $t, p>0,05$ ). Para as áreas superficiais dos ovos brancos, vermelhos e de codornas encontrados no modelo computacional, os valores médios e desvios padrões foram de $81,67 \pm 3,08 \mathrm{~cm}^{2}, 68,03 \pm 3,17 \mathrm{~cm}^{2} \mathrm{e}$ $21,88 \pm 1,32 \mathrm{~cm}^{2}$, respectivamente, enquanto que, para as áreas superficiais calculadas pela equação da literatura, os valores foram de $66,48 \pm 2,35 \mathrm{~cm}^{2}, 84,63 \pm 4,33$ $\mathrm{cm}^{2}$ e $25,09 \pm 1,53 \mathrm{~cm}^{2}$, respectivamente.

Diversos estudos na área de avicultura obtiveram os seguintes valores de $\mathrm{R}^{2}$ ao se comparar os valores 

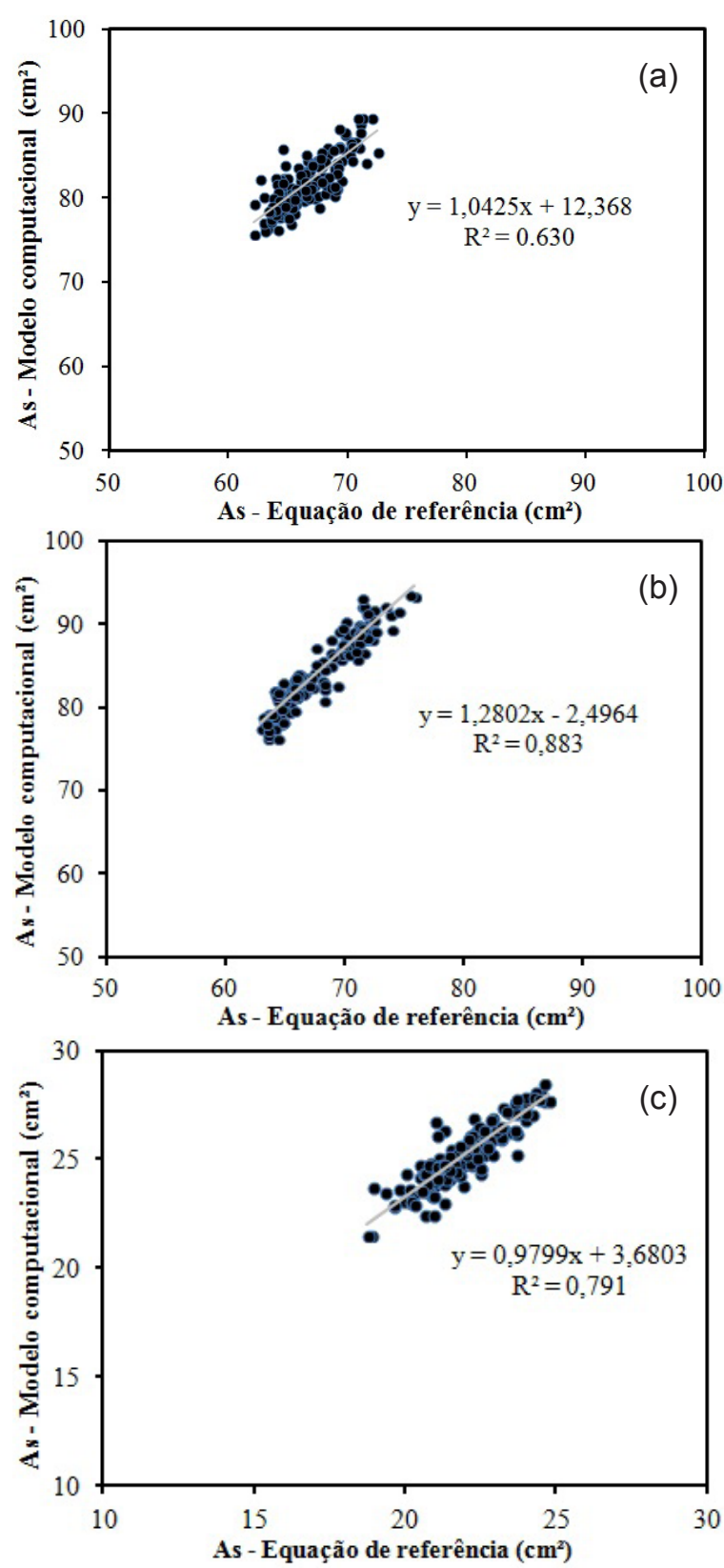

Figura 7. Comparação entre as áreas superficiais dos (a) ovos brancos, (b) vermelhos e (c) de codorna, observados pela modelo computacional e estimados pela equação de referência (Comparison between surface areas of (a) white, (b) red and (c) quail eggs, observed by computational model and estimated by the reference equation).

obtidos pelos modelos propostos aos experimentais: Castro et al. (2012), ao estimarem o peso de codornas japonesas, por meio de modelo fuzzy, ajustaram uma equação empírica para a estimativa da área superficial, encontrando $R^{2}$ de 0,668 . Silva et al. (2009), ajustou uma equação empírica para a estimativa da área superficial de frangos de corte em função da massa corporal, encontrando $R^{2}$ de 0,988 , evidenciando assim a eficiência do uso do coeficiente de determinação em testes e avaliações de novos modelos matemáticos.

De acordo com os dados experimentais de Silva (2014), ao avaliar a área superficial de ovos de galinha, encontrou valor médio de área superficial de $76,2 \mathrm{~cm}^{2}$. North and Bell (1990), investigando as características da forma do ovo, afirmam que o ovo padrão tem que abranger um volume de $63 \mathrm{~cm}^{3}$ e área superficial de $68 \mathrm{~cm}^{2}$, com peso aproximado de $56,7 \mathrm{~g}$. Com estes valores, o ovo se enquadra dentro da classificação grande descrita pela Resolução 05/91 do CIPOA/MAPA. Trindade et al. (2007), avaliando os parâmetros geométricos de ovos em galpões na região do semiárido paraibano, em condições de verão e inverno, encontraram valores médios de volume e área superficial de $64,60 \mathrm{~cm}^{3} \mathrm{e}$ $76,30 \mathrm{~cm}^{2}$, respectivamente. Mohsenin (1970) trabalhando com modelo elíptico encontrou valores médios para área superficial de $70,5 \mathrm{~cm}^{2}$.

Os valores médios dos volumes dos ovos brancos e vermelhos observados e calculados pela equação de referência encontrados neste estudo estão ligeiramentes abaixo do valor considerado como padrão. Entretanto, os valores médios dos ovos brancos e vermelhos determinados pelo método computacional estão próximos do valor padrão.

Correlacionando-se os valores de volume (V) e de área superficial (As) do método computacional em função do peso (P) dos ovos, conclui-se que o modelo matemático empírico que melhor se ajustou, em função do coeficiente de determinação, $\mathrm{R}^{2}$, foi o potencial, cujas equações são:

a) Ovos brancos

$$
\begin{array}{ll}
\mathrm{V}=4,2358 \times \mathrm{P}^{0,6923} & \mathrm{R}^{2}=0,519 \\
\mathrm{As}=11,502 \times \mathrm{P}^{0,4889} & \mathrm{R}^{2}=0,558
\end{array}
$$

b) Ovos vermelhos

$$
\begin{array}{ll}
\mathrm{V}=0,3746 \times \mathrm{P}^{1,2891} & \mathrm{R}^{2}=0,766 \\
\mathrm{As}=2,683 \times \mathrm{P}^{0,8462} & \mathrm{R}^{2}=0,761
\end{array}
$$

c) Ovos de codorna

$$
\begin{array}{ll}
\mathrm{V}=1,2964 \times \mathrm{P}^{0,9317} & \mathrm{R}^{2}=0,830 \\
\mathrm{As}=5,7478 \times \mathrm{P}^{0,6261} & \mathrm{R}^{2}=0,836
\end{array}
$$

Mohsenin (1970) encontrou o mesmo modelo empírico para estimar área de ovos, em que a constante da equação variou de 4,56 a 5,07, enquanto o expoente apresentou valor 0,66 . Trindade et al. (2007) também encontraram o mesmo modelo empírico para estimar volume e área superficial de ovos, sendo que as constantes das equações foram de 1,09 e 4,90, respectivamente, e expoente 0,99 e 0,67 , respectivamente.

\section{CONCLUSÕES}

Com base no exposto, pode-se concluir que o modelo matemático permitiu determinar o volume e área superficial de ovos, utilizando apenas três variáveis (comprimento, largura e peso) dos ovos que podem ser obtidos por meio de medidas simples.

Todos os modelos para estimativa do volume e da área superficial de ovos, propostos neste, apresentaram valores médios próximos aos obtidos pela equação usada como referência.

A obtenção do volume e da área supericial utilizando o peso dos ovos apresentou-se adequada e viável de ser aplicado aos ovos comerciais. Os maiores desvios nos valores de volume e área superficial ocorreram nos ovos brancos. 
Verificou-se que o modelo computacional pode ser devidamente aplicado para determinação do volume e área superficial de ovos, diminuindo o tempo de coleta manual dos dados avaliados. Ademais, a análise de imagem dos ovos mostrou-se adequada na determinação do volume e área superficial, apresentando-se como uma técnica não invasiva e não destrutiva adequada.

\section{AGRADECIMENTOS}

Os autores agradecem a Coordenação de Aperfeiçoamento de Pessoal de Nível Superior (CAPES), Conselho Nacional de Desenvolvimento Científico e Tecnológico (CNPq) e Fundação de Amparo à Pesquisa do Estado de Minas Gerais (FAPEMIG) pelo suporte financeiro ao projeto.

\section{BIBLIOGRAFIA}

Abreu, V.M.N. e Abreu, P.G. 2011. Os desafios da ambiência sobre os sistemas de aves no Brasil. Rev Bras Zootecn, 40: 1-14.

Bonnet, Y. e Mongin, P. 1965. Mesure de la surface de l'oeuf. (Measurement of egg shape). Ann Zootech, 14: 311-317.

Carioca, S.T.; Guimarães Cruz, F.G.; Matos, P.G.J.; Maquiné, L.C.; Chagas, E.O.; Oliveira, S.C. e Santos Filho, A.B. 2010. Influência dos níveis energéticos e protéicos em rações de poedeiras leves em Manaus. Arch Zootec, 59: 455-458.

Carvalho, L.S.S. e Fernandes, E.A. 2013. Formação e qualidade da casca de ovos de reprodutoras e poedeiras comerciais. Med Vet, 7: 35-44.

Castro, J.O.; Veloso, A.V.; Yanagi Junior, T.; Fassani, E.J.; Schiassi, L. e Campos, A.T. 2012. Estimate of the weight of japanese quail eggs through fuzzy sets theory. Ciênc Agrotec, 36: 108-116.

Malavazzi, G. 1995. Avicultura - Manual Prático. Ed. Nobel. São Paulo. 163 pp.

Mohsenin, N.N. 1970. Physical properties of plant and animal materials. Gordon and Breach Science Publishers. New York. 742 pp.
Narushin, V.G. 1993. New indestructive methods of egg parameters and eggshell quality determination. Fifth European Symposium on the Quality of Eggs and Egg Products. Anais... Tours. France. pp. 217-222.

Narushin, V.G. 1997. The avian egg: Geometrical description and calculation of parameters. J Agr Eng Res, 68: 201-205.

Narushin, V.G. 2001. Shape geometry of the avian egg. J Agr Eng Res, 79: $441-448$.

Narushin, V. G. 2005. Egg geometry calculation using the measurements of length and breadth. Poultry Sci, 84: 482-484.

Narushin, V.G.; Van Kempen, T.A.; Wineland, M.J. e Christensen, V.L. 2004. Comparing infrared spectroscopy and egg size measurements for predicting eggshell quality. Biosystems Eng, 87: 367-37.

North, M.O. e Bell, D.D. 1990. Commercial chicken production manual. 4th ed. An AVI Book. New York. 913 pp.

Preston, F.W. 1953. The shape of bird's eggs. The Auk, 70: 160-182.

Rehkugler, G.E. 1973. Characterizing the shape of a hen's egg. Poultry Sci, 52: 127-138.

Romanoff, A.L. e Romanoff, A.J. 1949. The avian egg. John Wiley. New York. NY. 918 pp.

SAS. 2001. SAS user's guide: statistical. Cary, NC. 438 pp.

Smart, I.H.M. 1967. The curve of the avian egg. J Anat, 101: 634-635.

Silva, E. 2014. Predição da área superficial e volume de ovos de galinhas poedeiras. Tese (Doutorado em Engenharia Agrícola). UFLA. Lavras. 61 pp.

Silva, E.; Yanagi Junior, T.; Braga Júnior, R.A.; Lopes, M.A.; Damasceno, F.A. e Silva, G.C.A. 2009. Desenvolvimento e validação de um modelo matemático para o cálculo da área superficial de frangos de corte. Eng Agr, 29: 1-7.

Silva, G.C.A.; Yanagi Junior, T.; Silva, E.; Braga Júnior, R. e Campos, A.T. 2011 . Recuperação da topografia de ovos por meio da Técnica de Moiré e calibração independente. Eng Agr, 31: 21 1-218.

Trindade, J.L.; Nascimento, J.W.B. e Furtado, D.A. 2007. Qualidade do ovo de galinhas poedeiras criadas em galpões no semi-árido paraibano. Rev Bras Eng Agr Ambiental, 11: 652-657.

Xavier, I.M.C.; Cansado, S.V.; Figueiredo, T.C.; Lara, L.J.C. Souza, M.R. e Baião, N.C. 2008. Qualidade de ovos de consumo submetidos a diferentes condições de armazenamento. Arq Bras Vet Zootec, 60: 953-959. 
